\title{
Ultrahigh Energy Cosmic-ray Accelerators
}

\author{
A. V. Olinto \\ Department of Astronomy \& Astrophysics \& Enrico Fermi Institute, \\ The University of Chicago, Chicago, IL 60637, U.S.A.
}

\begin{abstract}
The surprising lack of a high energy cutoff in the cosmic ray spectrum at the highest energies, together with an apparently isotropic distribution of arrival directions, have strongly challenged most models proposed for the acceleration of ultrahigh energy cosmic rays. Young neutron star winds may be able to explain the mystery. We discuss this recent proposal after summarizing the observational challenge and plausible acceleration sites. Young neutrons star winds differ from alternative models in the predictions for composition, spectrum, and angular distribution, which will be tested in future experiments.
\end{abstract}

\section{Introduction}

The detection of cosmic rays with energies above $10^{20} \mathrm{eV}$ has triggered considerable interest on the origin and nature of these particles. Many hundreds of events with energies above $10^{19} \mathrm{eV}$ and over a dozen events above $10^{20} \mathrm{eV}$ have now been observed by a number of experiments such as AGASA (Hayashida et al. 1994, Takeda et al. 1998, Takeda et al. 1999), Fly's Eye (Bird et al. 1993, 1994, 1995), and Haverah Park (Lawrence, Reid, \& Watson 1991). Most unexpected is the significant flux of events observed above $5 \times 10^{19} \mathrm{eV}$ (Takeda et al. 1998) with no sign of the Greisen-Zatsepin-Kuzmin (GZK) cutoff (Greisen 1966, Zatsepin \& Kuzmin 1966). A cutoff should be present if the ultrahigh energy particles are protons, nuclei, or photons from extragalactic sources. Cosmic ray protons of energies above $5 \times 10^{19} \mathrm{eV}$ lose energy to photopion production off the cosmic microwave background and cannot originate further than about $50 \mathrm{Mpc}$ away from Earth. Nuclei are photodisintegrated on shorter distances due to the infrared background (Puget, Stecker, \& Bredekamp 1976), while the radio background constrains photons to originate from even closer systems.

In addition to the presence of events past the GZK cutoff, there have been no clear counterparts identified in the arrival direction of the highest energy events. If these events are protons, they may point back to their sources within a few degrees, since at these high energies the Galactic and extra-galactic magnetic fields should not affect their orbits significantly. The gyroradius of a $10^{20} \mathrm{eV}$ proton is $100 \mathrm{kpc}$ in a $\mu$ Gauss field which is typical for the Galactic disk, therefore, protons propagate mainly in straight lines as they traverse the Galaxy. At present, no correlations between arrival directions and plausible optical counterparts such as sources in the Galactic plane, the Local Group, or the Local Supercluster have been found. The ultrahigh energy cosmic ray (UHECR) data is consistent with 
an isotropic distribution of sources, in contrast with the anisotropic distribution of light within $50 \mathrm{Mpc}$ of Earth.

\section{The UHECR Puzzle}

In attempting to explain the origin of UHECRs, models confront a number of challenges. The extreme energy is the greatest challenge that models for astrophysical acceleration face, and to complete the puzzle, models have to match the spectral shape, the primary composition, and the arrival direction distribution of the observed events.

If UHECRs are extragalactic, the observed highest energy event at $3 \times$ $10^{20} \mathrm{eV}$ (Bird 1994) argues for accelerators that reach as high as a $\mathrm{ZeV}(\mathrm{ZeV}$ $=10^{21} \mathrm{eV}$ ). The energetic requirements at the source increase with the distance traveled by the UHE primaries from source to Earth. Depending on the strength and structure of the magnetic field along the primary's path, the distance traveled may be significantly larger than the distance to the source. If $3 \times 10^{20} \mathrm{eV}$ is taken as a typical energy for protons travelling on straight lines, accelerators located further than $30 \mathrm{Mpc}$ need to reach above $1 \mathrm{ZeV}$, while those located further than $60 \mathrm{Mpc}$ require over $10 \mathrm{ZeV}$ (Cronin 1992). As magnetic fields above $\sim 10^{-8} \mathrm{G}$ may thread intragalactic space (Ryu \& Biermann 1998; Blasi, Burles, \& Olinto 1999), protons travel in curved paths, and sources need to be either more energetic or located closer to Earth.

The extreme energy requirements have encouraged alternative explanations for UHECRs. For early universe physics, a $\mathrm{ZeV}$ is not particularly high in energy. For instance, relics from the Grand Unified scale at $\sim 10^{24} \mathrm{eV}$ may be causing these ultrahigh energy events (Hill 1983; Schramm \& Hill 1983). The challenge for models that make use of early universe relics is generally the flux-the same challenge that observers face. At $10^{20} \mathrm{eV}$, the observed flux of UHECRs is about $\sim 1$ event $/ \mathrm{km}^{2} /$ century, which has strongly limited our ability to gather more than a dozen such events after decades of observations. Although challenging to observers and topological defect models, the flux is not particularly constraining in terms of general energetic requirements on astrophysical sources. In fact, this flux equals the flux of one gamma-ray burst that may have taken place in a $50 \mathrm{Mpc}$ radius volume around us (Waxman 1995; Vietri 1995).

The energy spectrum of cosmic rays has a steep energy dependence $N(E) \propto$ $E^{-\gamma}$, with $\gamma \approx 2.7$ between $\sim 10^{8} \mathrm{eV}$ and $\lesssim 10^{15} \mathrm{eV}$, and $\gamma \approx 3.1$ for $10^{15} \mathrm{eV}$ $\lesssim E \lesssim 10^{19} \mathrm{eV}$. Cosmic rays of energy up to $\sim 10^{15} \mathrm{eV}$ are widely accepted to originate in shocks associated with galactic supernova remnants, but this mechanism has difficulties producing particles of higher energy. The events with energy above $10^{19.5} \mathrm{eV}$, however, show a much flatter spectrum with $1 \lessgtr \gamma \lesssim 2$. The drastic change in slope suggests the emergence of a new component of cosmic rays at ultrahigh energies. This new component of cosmic rays is generally thought to be extragalactic, but they may also originate in an extended halo or in the Galaxy (Olinto, Epstein, \& Blasi 1999), depending on their composition.

The observed spectrum represents a convolution of the source primary spectrum with the effect of energy losses during the propagation between the source and the Earth. If the new component is extragalactic, loss processes modify the spectral shape significantly. For primary protons, the main loss processes 
are pair production (Blumenthal 1970) and photopion production off the cosmic microwave background radiation (Greisen 1966; Zatzepin \& Kuzmin 1966). For straight line propagation, loss processes limit sources of $10^{20} \mathrm{eV}$ to be within about $50 \mathrm{Mpc}$ from us. For heavier nuclei, the infrared background induces losses (Stecker 1999), and the maximum distance for $10^{20} \mathrm{eV}$ nuclei to originate from is $\sim 10 \mathrm{Mpc}$. In the case of photon primaries, the radio background represents the main source of losses, and the distance is limited to $\lesssim 10 \mathrm{Mpc}$. Depending on the poorly known strength and structure of the extragalactic magnetic fields, the GZK cutoff moves closer in distance for charged primaries. In addition, plausible models have to accommodate the spectrum at the highest energies $\left(Z 10^{20} \mathrm{eV}\right)$ without overproducing cosmic rays at $\gtrsim 10^{18} \mathrm{eV}$ (Berezinsky, Grigorieva, \& Dogiel 1989; Blasi \& Olinto 1998; Sigl, Lemoine, \& Biermann 1999).

Charged particles of energies up to $10^{20} \mathrm{eV}$ can be deflected significantly in cosmic magnetic fields. The Larmor radius of a particle with energy $E$ and charge $Z e$ in a magnetic field $B$ is $r_{L}=0.1 \mathrm{Mpc}\left(E / 10^{20} \mathrm{eV}\right) / Z(B / \mu \mathrm{G})$. If the UHECR primaries are protons, only large-scale intergalactic magnetic fields affect their propagation significantly, while for heavier nuclei, the Galactic magnetic field should also be taken into account. While the Galactic magnetic field is reasonably well studied, extragalactic fields are still very poorly understood.

Together with a composition determination, the distribution of arrival directions can hold the key to the UHECR puzzle. Within a $50 \mathrm{Mpc}$ radius volume around us, the most luminous structures are the Galactic plane, the Local Group, and the general galaxy distribution with a relative overdensity around the region of the Local Supercluster. If the UHECR source is dark matter, then the Galactic Halo is the relevant structure which is expected to be a spheroidal overdensity centered around the Galaxy. On larger scales, the dark matter distribution should correlate with the luminous matter distribution. For the few highest energy events, there is presently no strong evidence for correlations between the events' arrival direction and any of these known structures (Stanev 1999): the distribution is isotropic to first approximation. Since the number of observed events above $10^{20} \mathrm{eV}$ is low, it is still too early to tell. For slightly lower energies, some correlations have been detected. Recently, the AGASA group has announced that the distribution of $10^{18} \mathrm{eV}$ events shows a significant correlation with the Galactic Center and the nearby Galactic spiral arms (Yoshida et al. 1999). If these correlations are confirmed, it would be strong evidence for a Galactic origin for cosmic rays around $10^{18} \mathrm{eV}$.

\section{UHECR Accelerators}

As mentioned above, there are great difficulties with finding plausible accelerators for such extremely energetic particles. Even the most powerful astrophysical objects such as radio galaxies and active galactic nuclei can barely accelerate charged particles to energies as high as $10^{20} \mathrm{eV}$.

Acceleration of UHECRs in astrophysical plasmas occurs when large-scale macroscopic motion, such as shocks and turbulent flows, is transferred to individual particles. The maximum energy, $E_{\max }$, is usually estimated by requiring that the gyroradius of the particle be contained in the acceleration region. Therefore, $E_{\max }$ is usually associated with the strength, $B$, and coherence length, 


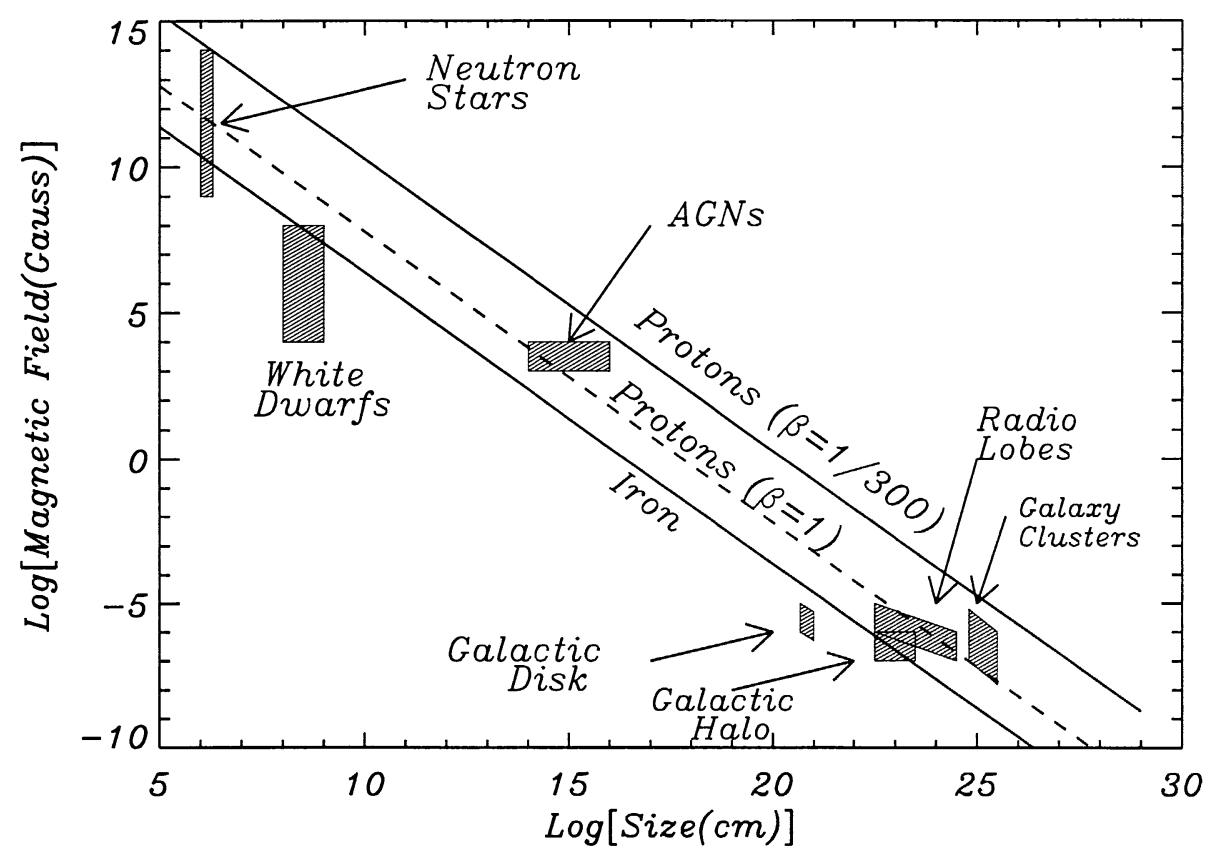

Figure 1. $B$ vs. $L$ for $E_{\max }=\beta Z e B L$.

$L$, of the magnetic field embedded in the plasma, such that $E_{\max } \sim \beta Z e B L$, where usually $\beta \sim v / c$, and $Z e$ is the charge of the particle. As can be seen in Figure 1, for $E_{\max } \sim 10^{20} \mathrm{eV}$ and $Z \beta \sim 1$, the only known astrophysical sources with reasonable $B L$ products are neutron stars $\left(B \sim 10^{13}\right.$ Gauss and $L \sim 10 \mathrm{~km})$, active galactic nuclei (AGNs) $\left(B \sim 10^{4}\right.$ Gauss and $\left.L \sim 10 \mathrm{AU}\right)$, radio lobes of active galaxies ( $B \sim 10^{-5}$ Gauss and $L \sim 10 \mathrm{kpc}$ ), and clusters of galaxies ( $B \sim 10^{-6}$ Gauss and $L \sim 100 \mathrm{kpc}$ ) (Hillas 1984).

In general, when these sites are considered more carefully, one finds great difficulties due to either energy losses in the accelerating regions or the great distances of known sources from our galaxy. In most of these objects, one invokes shock acceleration as the primary acceleration mechanism. As discussed by Achterberg in this meeting, shock acceleration is not effective in reaching $\mathrm{ZeV}$ energies for most proposed accelerators, with the possible exception of galaxy cluster shocks. The problem with clusters of galaxies as sources of UHECRs on Earth is that our location in the universe is not close enough to a cluster shock to avoid the GZK cutoff. Furthermore, UHECRs generated in typical clusters do not escape from them during the age of the universe (Blasi \& Olinto 1998).

Moving left on Figure 1, radio lobes in FRII objects can reach the required energy if $\beta \sim 1$ (Rachen \& Biermann 1993). Again, location is a challenge since these are rare objects and far apart. A recent proposal places the source of all UHECRs observed in a single object, M87, by invoking a Galactic wind that can make different observed arrival directions trace back to M87 (Ahn et al., in 
preparation). The existence of a Galactic wind with the required characteristics to allow for this possibility is yet to be determined.

Active galactic nuclei (AGNs) are powerful engines where matter accretes onto very massive black holes. The problem with AGNs as UHECR sources is two-fold: one is the distance to more active objects, and the other is common among highly energetic environments-losses due to intense radiation field downgrade particle energies well below the maximum achievable energy. These limitations have led to the proposal that remnant quasars, large black holes in the centers of inactive galaxies, are UHECR accelerators (Boldt \& Ghosh 1999). In this case, one would have no obvious counterpart, since any galaxy would be equally likely to host such accelerators and losses are not as significant. The spectrum will be dominated by the local distribution of galaxies, with more distant galaxies inducing a GZK cutoff (see, e.g., Medina-Tanco 1999). The detailed acceleration mechanism for this proposal is yet to be determined.

Before discussing the last possibility in Figure 1, neutron stars, it is worth mentioning that the lack of a clear astrophysical solution for the UHECR puzzle has produced a number of models based on physics beyond the standard model, such as monopoles, cosmic strings, cosmic necklaces, vortons, and superheavy long-lived decaying relic particles, to name a few. We refer the interested reader to a few recent reviews (Berezinsky 1999; Bhattacharjee \& Sigl 2000).

\subsection{Young Neutron Star Winds}

As shown in Figure 1, neutron stars may be effective in accelerating UHECRs (see, e.g., Berezinsky et al. 1990). Acceleration processes inside the neutron star light cylinder are bound to fail much like the AGN case: ambient magnetic and radiation fields induce significant losses (Venkatesan, Miller, \& Olinto 1997). However, the plasma that expands beyond the light cylinder is free from the main loss processes and may be accelerated to ultrahigh energies.

One possible solution to the UHECR puzzle is our recent proposal that the early evolution of neutron stars may be responsible for the unexplained flux of cosmic rays beyond the GZK cutoff (Olinto, Epstein, \& Blasi 1999; Blasi, Epstein, \& Olinto 2000). In this case, UHECRs originate in our galaxy and are due to iron nuclei accelerated from the surface of strongly magnetic, young neutron stars.

Newly formed, rapidly rotating neutron stars may accelerate iron nuclei to ultrahigh energies through relativistic MHD winds beyond their light cylinders (see, e.g., Michel 1991). The nature of the relativistic wind is not yet clear, but observations of the Crab Nebula indicate that most of the rotational energy emitted by the pulsar is converted into the flow kinetic energy of the particles in the wind. If most of the magnetic energy in the wind zone is converted into particle kinetic energy and the rest mass density of the wind is not dominated by electron-positron pairs, particles in the wind can reach a maximum energy of $E_{\max } \simeq 8 \times 10^{20} Z_{26} B_{13} \Omega_{3 k}^{2} \mathrm{eV}$ for iron nuclei $\left(Z_{26} \equiv Z / 26=1\right)$, neutron star surface fields $B=10^{13} B_{13}$ Gauss, and initial rotation frequency $\Omega=3000 \Omega_{3 k} \mathrm{~s}^{-1}$. In the rest frame of the wind, the plasma is relatively cold, while in the star's rest frame, the plasma moves with Lorentz factors $\gamma \sim 10^{9}-10^{10}$.

The typical energy of the accelerated cosmic rays can be estimated by considering the magnetic energy per ion at the light cylinder $E_{\mathrm{cr}} \simeq B_{\mathrm{lc}}^{2} / 8 \pi n_{\mathrm{GJ}}$ where 


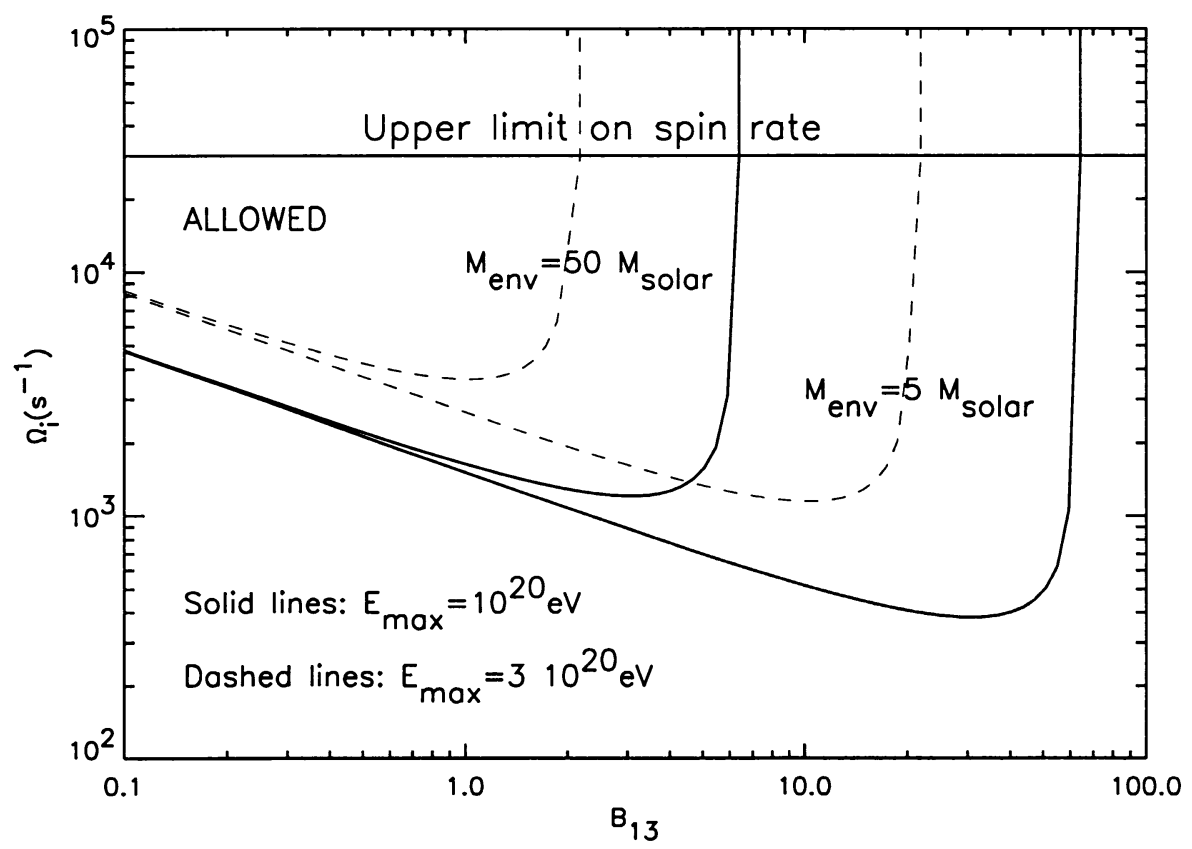

Figure 2. Solid lines for $E_{\mathrm{cr}}=10^{20} \mathrm{eV}$ and dashed lines for $E_{\mathrm{cr}}=$ $3 \times 10^{20} \mathrm{eV}$. The curves are plotted for two values of the envelope mass, $M_{\text {env }}=50 M_{\text {solar }}$ and $M_{\text {env }}=5 M_{\text {solar }}$, as indicated. The horizontal line at spin period $\sim 0.3 \mathrm{~ms}$ indicates the minimum period allowed for neutron stars.

the Goldreich-Julian (1969) density is $n_{\mathrm{GJ}}=1.7 \times 10^{11} B_{13} \Omega_{3 k}^{4} / Z \mathrm{~cm}^{-3}$. We find $E_{\text {cr }} \simeq 4 \times 10^{20} Z_{26} B_{13} \Omega_{3 k}^{2} \mathrm{eV}$, similar to $E_{\max }$ above. Therefore, neutron stars whose initial spin periods are shorter than $\sim 4\left(B_{S} / 10^{13} \mathrm{G}\right) \mathrm{ms}$ can accelerate iron nuclei to greater than $10^{20} \mathrm{eV}$.

About a year after the supernova explosion, the iron nuclei can escape through the remnant of the supernova that produced the neutron star, without suffering significant spallation. The supernova event ejects the envelope of the original star, making it possible for cosmic rays to escape. However, as the envelope expands, the young neutron star spins down and may become unable to emit particles of the necessary energy. A requirement for relativistic winds to supply UHECRs is that the column density of the envelope becomes transparent to UHECR iron before the spinning rate of the neutron star decreases significantly. The allowed parameter space for this model is shown in Figure 2.

The spectrum of accelerated UHECRs is determined by the evolution of the rotational frequency: as the star spins down, the energy of the cosmic ray particles ejected with the wind decreases. The predicted spectrum is very flat, $\gamma=1$, in agreement with the UHECR data. Furthermore, for the parameters within the allowed region, the acceleration and survival of UHECR iron nuclei is not significantly affected by the ambient photon radiation. 
Depending on the structure of the galactic magnetic field, the trajectories of the iron nuclei from galactic sources can be consistent with the observed arrival directions of the highest energy events (Zirakashvili et al. 1998). The gyroradius of these UHECRs in the Galactic field of strength $B_{\text {gal }}=3 \mu$ Gauss is $r_{B}=1.4\left(E_{20} / Z_{26}\right) \mathrm{kpc}$, which is considerably less than the typical distance to a young neutron star $(\sim 8 \mathrm{kpc})$. Furthermore, the cosmic ray component at $10^{18} \mathrm{eV}$ is nearly isotropic. If these cosmic rays are protons of Galactic origin, the isotropic distribution observed at these energies may be indicative of the diffusive effect of the Galactic and halo magnetic fields. Iron at $10^{20} \mathrm{eV}$ probes similar trajectories to protons at a few times $10^{18} \mathrm{eV}$.

\section{Conclusion}

Future experiments such as the Auger Project and the OWL-Airwatch satellite will be able to discriminate between different models (Cronin 1999; Watson 2000). An excellent discriminator would be an unambiguous composition determination of the primaries. In general, Galactic disk models invoke iron nuclei in order to be consistent with the isotropic distribution of events, and extragalactic astrophysical models tend to favor proton primaries, while photon primaries are more common for early universe relics. The observational tools in place for composition discrimination are the muon content of shower in the ground arrays (more muons for nuclei vs. nucleons) and the depth of shower maximum in fluorescence detectors (the heavier the primary, the deeper in the atmosphere their shower maximum is). In addition, the correlation of arrival directions for events with energies above $10^{20} \mathrm{eV}$ with some known structure would be key in differentiating between different models. For example, we should see a correlation with the Galactic center and disk for the case of young neutron star winds (see Stanev 1999; Yoshida et al. 1999), and the large scale galaxy distribution for the case of quasar remnants. Both aspects will be testable with future experiments.

Acknowledgments. We thank the organizers and acknowledge the support of NSF through grant AST 94-20759 and DOE through grant DE-FG0290ER40606 at the University of Chicago.

\section{References}

Ahn, E. J., Biermann, P. L., Medina-Tanco, G., \& Stanev, T., in preparation Berezinsky, V. S. 1999, Nucl. Phys. (Proc. Suppl.), 70, 419

Berezinsky, V. S., Bulanov, S. V., Dogiel, V. A., Ginzburg, V. L., \& Ptuskin, V. S. 1990, Astrophysics of Cosmic Rays ed. V. L. Ginzburg (Amsterdam: North Holland)

Berezinsky, V. S., Grigo'eva, S. I., \& Dogiel, V. A. 1989, Sov. Phys. JETP, 69, 453

Bhattacharjee, P., \& Sigl, G. 2000, Phys. Reps., submitted

Bird, D. J. 1993, Phys. Rev. Lett., 71, 3401

Bird, D. J. 1994, ApJ, 424, 491

Bird, D. J., et al. 1995, ApJ, 441, 144 
Blasi, P., Burles, S., \& Olinto, A. V. 1999, ApJ, 512, L79

Blasi, P., Epstein, R. I., \& Olinto, A. V. 2000, Phys. Rev. Lett., submitted

Blasi, P., \& Olinto, A. V. 1998, Phys. Rev. D, 59, 023001

Blumenthal, G. B. 1970, Phys. Rev. D, 1, 1596

Boldt, E., \& Ghosh, P. 1999, MNRAS, 307, 491

Cronin, J. W. 1992, Nucl. Phys. B. (Proc. Suppl.), 28, 213

Cronin, J. W. 1999, Rev. Mod. Phys., 71, S165

Goldreich, P., \& Julian, W. H. 1969, ApJ, 157, 869

Greisen, K. 1966, Phys. Rev. Lett., 16, 748

Hayashida, N. 1994, Phys. Rev. Lett., 73, 3491

Hill, C. T. 1983, Nucl. Phys. B, 224, 469

Hillas, A. M. 1984, ARA\&A, 22, 425

Lawrence, M. A., Reid, R. J. O., \& Watson, A. A. 1991, J. Phys. G: Nucl. Part. Phys., 17, 773

Medina-Tanco, G. 1999, in Proc. of 26th ICRC, eds. D. Kieda, M. Salamon, \& B. Dingus (Salt Lake City: University of Utah), 4, 346

Michel, F. C. 1991, Theory of Neutron Stars Magnetosphere (Chicago: The University of Chicago Press)

Olinto, A. V., Epstein, R. I., \& Blasi, P. 1999, in Proc. of 26th ICRC, eds. D. Kieda, M. Salamon, \& B. Dingus (Salt Lake City: University of Utah), 4,361

Puget, J. L., Stecker, F. W., \& Bredekamp, J. H. 1976, ApJ, 205, 638

Rachen, J. P., \& Biermann, P. L. 1993, A\&A, 272, 161

Ryu, D., \& Bierman, P. L. 1998, A\&A, 335, 19

Schramm, D. N., \& Hill, C. T. 1983, in Proc. of 18th ICRC, 2, 393

Sigl, G., Lemoine, M., \& Biermann, P. 1999, Astropart. Phys., 10, 141

Stanev, T., \& Hillas, A. M. 1999, in Proc. of 26th ICRC, eds. D. Kieda, M. Salamon, \& B. Dingus (Salt Lake City: University of Utah), 4, 365

Stecker, F. W. 1999, Astropart. Phys., 11, 83

Takeda, M., et al. 1998, Phys. Rev. Lett., 81, 1163

Takeda, M., et al. 1999, ApJ, 522, 225

Venkatesan, A., Miller, M. C., \& Olinto, A. V. 1997, ApJ, 484, 323

Vietri, M. 1995, ApJ, 453, 883

Watson, A. A. 2000, Phys. Reps., submitted

Waxman, E. 1995, Phys. Rev. Lett., 75, 386

Yoshida, S., et al. (AGASA Collaboration) 1999, in Proc. of 26th ICRC, eds. D. Kieda, M. Salamon, \& B. Dingus (Salt Lake City: University of Utah), 3,256

Zatsepin, G. T., \& Kuzmin, V. A. 1966, Sov. Phys. JETP Lett., 4, 78

Zirakashvili, V. N., Pochepkin, D. N., Ptuskin, V. S., \& Rogovaya, S. I. 1998, Astron. Lett., 24, 139 